\title{
Correction to: Ornamental plants adapted to urban ecosystem pollution: lawn grasses and painted daisy tolerating copper
}

\author{
Evgeny Aleksandrovich Gladkov ${ }^{1}\left[\right.$ [ Ilina Igorevna Tashlieva ${ }^{1}$ (D) $\cdot$ Olga Victorovna Gladkova $^{2}$ (I)
}

Published online: 27 October 2021

(c) Springer-Verlag GmbH Germany, part of Springer Nature 2021

Correction to: Environmental Science and Pollution Research (2021) 28:14115-14120

https://doi.org/10.1007/s11356-020-11423-6

\section{Author contributions}

Evgeny Aleksandrovich Gladkov-46\%

Ilina Igorevna Tashlieva-30\%

Olga Victorovna Gladkova-24\%

Publisher's Note Springer Nature remains neutral with regard to jurisdictional claims in published maps and institutional affiliations.

The original article can be found online at https://doi.org/10.1007/ s11356-020-11423-6.

Evgeny Aleksandrovich Gladkov

gladkovu@mail.ru

$\triangle$ Ilina Igorevna Tashlieva

ii_tash@mail.ru

$\bowtie$ Olga Victorovna Gladkova

olgav.gladkova@mail.ru

1 K.A. Timiryazev Institute of Plant Physiology RAS, IPP

RAS, 35, Botanicheskaya St., Moscow 127276, Russia

2 Independent Scientist, Prospekt Mira, 181, Moscow, Russia 